\title{
Antimicrobial susceptibility of Neisseria gonorrhoeae isolates from symptomatic men attending the Nanjing sexually transmitted diseases clinic (2011-2012): genetic characteristics of isolates with reduced sensitivity to ceftriaxone
}

\author{
Sai Li ${ }^{1}$ Xiao-Hong Su ${ }^{*}$, Wen-Jing Le' ${ }^{1}$ Fa-Xing Jiang ${ }^{2}$, Bao-Xi Wang ${ }^{1}$ and Peter A Rice ${ }^{3}$
}

\begin{abstract}
Background: Evolving gonococcal antimicrobial resistance (AMR) poses a serious threat to public health. The aim of this study was to: update antimicrobial susceptibility data of Neisseria gonorrhoeae recently isolated in Nanjing, China and identify specific deteminants of antimicrobial resistance and gentoypes of isolates with decreased sensitivity to ceftriaxone.

Methods: $334 \mathrm{~N}$. gonorrhoeae isolates were collected consecutively from symptomatic men attending the Nanjing STD Clinic between April 2011 and December 2012. The minimum inhibitory concentrations (MICs) for penicillin, tetracycline, ciprofloxacin, spectinomycin and ceftriaxone were determined by agar plate dilution for each isolate. Penicillinase-producing N. gonorrhoeae (PPNG) and tetracycline-resistant N. gonorrhoeae (TRNG) were examined and typed for $\beta$-lactamase and tetM encoding plasmids respectively. Isolates that displayed elevated MICs to ceftriaxone ( $\mathrm{MIC} \geq 0.125 \mathrm{mg} / \mathrm{L}$ ) were also tested for mutations in penA, mtrR, porB1b, ponA and pilQ genes and characterized by Neisseria gonorrhoeae multi-antigen sequence typing (NG-MAST).
\end{abstract}

Results: $98.8 \%$ (330/334) of $N$. gonorrhoeae isolates were resistant to ciprofloxacin; $97.9 \%$ (327/334) to tetracycline and $67.7 \%(226 / 334)$ to penicillin. All isolates were susceptible to ceftriaxone (MIC $\leq 0.25 \mathrm{mg} / \mathrm{L})$ and spectinomycin (MIC $\leq 32 \mathrm{mg} / \mathrm{L}$ ). Plasmid mediated resistance was exhibited by 175/334 (52\%) of isolates: 120/334 (36\%) of isolates were PPNG and 104/334 (31\%) were TRNG. 90.0\% (108/120) of PPNG isolates carried the Asia type $\beta$-lactamase encoding plasmid and 96\% (100/104) of TRNG isolates carried the Dutch type tetM containing plasmid. Elevated MICs for ceftriaxone were present in 15 (4.5\%) isolates; multiple mutations were found in penA, mtrR, porB1b and ponA genes. The 15 isolates were distributed into diverse NG-MAST sequence types; four different non-mosaic penA alleles were identified, including one new type.

Conclusions: N. gonorrhoeae isolates in Nanjing generally retained similar antimicrobial resistance patterns to isolates obtained five years ago. Fluctuations in resistance plasmid profiles imply that genetic exchange among gonococcal strains is ongoing and is frequent. Ceftriaxone and spectinomycin remain treatments of choice of gonorrhea in Nanjing, however, decreased susceptibility to ceftriaxone and rising MICs for spectinomycin of N. gonorrhoeae isolates underscore the importance of maintaining surveillance for AMR (both phenotypic and genotypic).

Keywords: Neisseria gonorrhoeae, Antimicrobial resistance, Resistance plasmids, Ceftriaxone, Resistance determinants

\footnotetext{
* Correspondence: suxh@ncstdlc.org

${ }^{1}$ STD Clinic, Institute of Dermatology, Chinese Academy of Medical Sciences and Peking Union Medical College, Nanjing 210042, China

Full list of author information is available at the end of the article
} 


\section{Background}

Gonorrhea is the fifth most commonly reported infectious disease in China; nearly 100,000 cases were reported officially nationwide in 2013 [1]. Worldwide, over 106 million new infections are diagnosed annually [2]. Effective treatment is key in preventing complications and controlling transmission of $N$. gonorrhoeae. Unfortunately, this bacterium has developed resistance to antimicrobials (AMR) including sulfonamides, penicillins, tetracyclines and quinolones $[3,4]$. In recent years treatment failures have occurred when extended-spectrum cephalosporins (ESCs), the most widely used agents for treatment of gonorrhea, were used, and associated increases in AMR to ESCs have been reported in several countries [5-11]. The molecular mechanisms associated with reduced susceptibility or resistance to ESCs include mutations in several gene loci; penA [12-16], mtrR $[17,18]$, penB $[17,19]$, ponA $[6,20]$ and pilQ $[21,22]$ and their multifaceted interactions $[6,23]$.

Several public health organizations have published their action/response plans to control the spread and impact of ESCs-resistant gonococci $[2,24,25]$. These have stressed the importance of enhancing quality-assured surveillance of gonococcal AMR both locally and internationally, with a focus on ESCs.

In China, the surveillance of $N$. gonorrhoeae AMR is conducted by the National Center for Sexually Transmitted Diseases (STDs) Control, China CDC, which systematically collects $N$. gonorrhoeae AMR data from $10 \sim 16$ geographically separate monitoring sentinels and provides external quality assessment. Annual AMR rates are available in brief reports issued by China CDC (1987-current) [26] and are also published by the World Health Organization (WHO), Western Pacific Region Gonococcal Antimicrobial Surveillance Programme (1992-current) [27-29].

During 2011-2012, the period of this investigation, ceftriaxone and spectinomycin were the only antimicrobial agents recommended officially in the Chinese guidelines for the treatment of gonorrhea [30], however, a wide variety of antimicrobials including (fluoro) quinolones were prescribed unofficially outside of STD Clinics and purchased over the counter to treat gonorrhea.

The Nanjing STD clinic, operating as a sentinel site in Eastern China, performs AMR tests routinely on $10 \%-20 \%$ of gonococcal isolates recovered in this city. We performed this study in 2011-12, to assess the impact, in Nanjing, of recent changes in ceftriaxone susceptibility of $N$. gonorrhoeae that have been reported [31-34] and to characterize genetic determinants and NG-MAST sequence types (STs) of isolates with decreased sensitivity to ceftriaxone.

\section{Methods}

\section{Subjects and bacterial strains}

$N$. gonorrhoeae isolates were isolated from male adults with symptoms/signs of urethritis (confirmed by $\geq 5$ polymorphonuclear neutrophils [PMNs]/oil immersion microscopic field seen in all urethral specimens) in the STD clinic of the National Center for STD Control in Nanjing, China between April 2011 and December 2012. These isolates represented $18.1 \%$ of the reported gonorrhea cases in Nanjing during this period. Epidemiological data was acquired by interviews conducted by clinical staff using standardized data recording instruments. Isolates from all but 2 men were from ethnic Han (332/334) and 99.4\% (332/ $334)$ of men were heterosexual. The mean ( \pm SEM) age was $36.7 \pm 3.3$ years, the median, 36 (range: 19 to 65), there being no significant difference $(p>0.05)$ compared with the national average age of gonorrhea cases in men (mean $[ \pm \mathrm{SEM}], 34.9 \pm 3.2$ ). $61.3 \%$ of men were married. Approval to enroll human subjects was given by the Institutional Review Board of the Institute of Dermatology, Chinese Academy of Medical Science. The study was introduced and explained to potential subjects who voluntarily gave written and signed consent. Urethral specimens were collected with cotton swabs and immediately streaked onto Thayer-Martin (T-M) selective medium. Inoculated plates were incubated at $36^{\circ} \mathrm{C}$ in candle jars for 24-48 h. N. gonorrhoeae was identified by colonial morphology, Gram's stain, and oxidase testing, which are sufficient to identify $N$. gonorrhoeae colonies isolated on selective medium, particularly for samples from the urethral tracts of symptomatic men [35,36]. Isolates were subcultured onto GC agar base (Difco, Detroit, MI) supplemented with $1 \%$ Isovitale $\mathrm{X}^{\mathrm{sm}}$ (Oxoid, USA) and pure cultures swabbed, suspended in tryptone-based soy broth and frozen $\left(-70^{\circ} \mathrm{C}\right)$ until used for antimicrobial testing.

\section{Antimicrobial susceptibility testing}

The minimum inhibitory concentrations (MICs; mg/L) of $N$. gonorrhoeae to penicillin, tetracycline, ciprofloxacin, spectinomycin and ceftriaxone were determined by the agar dilution method (Clinical Laboratory Standards Institute [37]), using 2-fold serial dilutions of antibiotics (all purchased from Sigma Aldrich, Saint Louis, MO, USA): penicillin $0.125-8 \mathrm{mg} / \mathrm{L}$, tetracycline $0.25-16 \mathrm{mg} / \mathrm{L}$, ciprofloxacin $0.25-8 \mathrm{mg} / \mathrm{L}$, spectinomycin $4-128 \mathrm{mg} / \mathrm{L}$ and ceftriaxone $0.004-0.5 \mathrm{mg} / \mathrm{L}$. WHO reference strains A, G, I and J (kindly provided by Dr. J. W. Tapsall, Prince of Wales Hospital, Sydney, Australia) and a ceftriaxoneresistant strain (kindly provided by Carmen Ardanuy, L'Hospitalet de Llobregat, Barcelona, Spain [38]) were used as controls. In addition, we included, as controls, two strains isolated from our prior study that had diminished susceptibiltiy to ceftriaxone (MIC $=0.125$ and $0.25 \mathrm{mg} / \mathrm{L}$ ) [39]. MIC breakpoints for assigning susceptibility, intermediate and resistance status to $N$. gonorrhoeae isolates were determined according to Clinical and Laboratory Standards Institute (CLSI) Document M07-A9 (M100S22) [37]. 
Production of $\beta$-lactamase was identified by paper acidometric test (phenotype method) [39,40]. Resistance patterns were defined as follows [41]:

- PPNG (Penicillinase-producing N. gonorrhoeae), $\beta$-lactamase positive

- TRNG (Plasmid-mediated tetracycline-resistant $N$. gonorrhoeae), MIC to tetracycline $\geq 16 \mathrm{mg} / \mathrm{L}$, also confirmed by PCR-based genotyping

- PPNG/TRNG, $\beta$-lactamase positive and MIC to tetracycline $\geq 16 \mathrm{mg} / \mathrm{L}$

- PenR (chromosomally mediated resistance to penicillin), non-PPNG, non-TRNG, MIC to penicillin $\geq 2 \mathrm{mg} / \mathrm{L}$ and $\mathrm{MIC}$ to tetracycline $<2 \mathrm{mg} / \mathrm{L}$

- TetR (chromosomally mediated resistance to tetracycline), non-PPNG, non-TRNG, MIC to tetracycline $\geq 2 \mathrm{mg} / \mathrm{L}$ and $\mathrm{MIC}$ to penicillin $<2 \mathrm{mg} / \mathrm{L}$

- CMRNG (chromosomal resistance to penicillin and tetracycline), non-PPNG, non-TRNG, MIC to penicillin $\geq 2 \mathrm{mg} / \mathrm{L}$ and $\mathrm{MIC}$ to tetracycline $\geq 2 \mathrm{mg} / \mathrm{L}$.

\section{Identification and typing of tet $M$ and $\beta$-lactamase gene producing plasmids}

Bacterial DNA was extracted according to a published method [39].

For tet $M$ gene containing plasmids, PCR reactions were performed using a universal forward primer; $5{ }^{\prime 825} \mathrm{CTCG}$ AACAAGAGGAAAGC ${ }^{842}$ and reverse primers $5^{, 1602} \mathrm{GC}$ ATTCCACTTCCCAAC ${ }^{1586}$ to identify the presence of the "American" type tet $M$ plasmid and 5' ${ }^{1267}$ TGCAGC AGAGGGAGG ${ }^{1253}$ to identify the "Dutch" type. PCR products were separated by agarose electrophoresis, stained with ethidium bromide and visualized using ultraviolet fluorescent light for the presence of a $778 \mathrm{bp}$ fragment ("American") or a 443 bp fragment ("Dutch") [42]. An American type tet $M$ containing strain identified in our previously study [39] and WHO reference strain G (Dutch type) were used as positive controls.

$\beta$-lactamase producing plasmids were characterized using multiplex PCR (employing specific forward and reverse primers [43]) followed by elecrophoresis to identify specifically sized PCR products, thereby differentiating three common plasmids: Asian (958 bp), African (1191 bp) and Toronto (650 bp). Positive control PCR products derived from plasmids pJD4 (Asian type), pJD5 (African type) and pJD7 (Toronto type) (provided by J.R. Dillon, University of Saskatchewan, Canada) were included in each assay.

\section{Sequencing of $N$. gonorrhoeae genetic determinants} associated with decreased susceptibility to ceftriaxone Amplification of penA, $m \operatorname{tr} R, \operatorname{por} B$ (and also pon $A$ and pilQ) was performed using published primers and conditions $[44,45]$. PCR products were sequenced twice bidirectionally on an Applied Biosystems 3730XL DNA automatic sequencer. Nucleotide and deduced amino acid sequences were edited and aligned against their respective prototypes [44]. A newly identified PBP2 type was designated XLI according to previously used nomenclature [12,15,46-49].

\section{Molecular epidemiological typing}

$N$. gonorrhoeae multiantigen sequence typing (NG-MAST) was performed by PCR amplification and sequencing of two high polymorphic fragments in $\operatorname{por} B$ and $t b p B$ genes according to previously described methods [50]. Allele numbers and sequence type numbers were assigned through the NG-MAST database (http://www.ng-mast.net).

\section{Nucleotide sequence accession numbers}

por $B$ sequences were submitted to the GenBank nucleotide database under accession numbers [Genbank: KF660589KF660594] and [Genbank: KF668353- KF668360]. Novel penA nucleotide sequences associated with reduced susceptibility to ESCs identified in this study were deposited in the GenBank database under accession number [Genbank: KF576657].

\section{Statistical analysis}

The Chi-Square test (2-tailed) was used to assess significance of associations and compare resistance rates; $\mathrm{p}$ values $<0.05$ were considered significant. Statistical analyses were performed using IBM SPSS Statistics version 22.0 .

\section{Results}

\section{Antimicrobial susceptibilities}

Among 652 consecutively enrolled male subjects with urethritis, 340 (52.1\%) were infected with N. gonorrhoeae; antimicrobial susceptibility testing of 334 successfully retrieved isolatesis is summarized in Table 1 (six isolates were not recovered from storage or could not be separated from contaminants). Resistance rates to antibiotics formerly or now recommended for treatment of gonorrhea in China were: ciprofloxacin 98.8\%, tetracycline $97.9 \%$, penicillin $67.7 \%$, all isolates were susceptible to spectinomycin and ceftriaxone. The minimum inhibitory concentration of $50 \%$ and $90 \%$ of gonococcal isolates $\left(\mathrm{MIC}_{50} / \mathrm{MIC}_{90}\right)$ for ciprofloxacin, tetracycline, penicillin, spectinomycin and ceftriaxone were $>8 />8$, $8 />16,2 />8,16 / 32$ and $0.03 / 0.06 \mathrm{mg} / \mathrm{L}$, respectively.

\section{Penicillin and tetracycline resistance phenotypes}

Penicillin and tetracycline resistance phenotypes were categorized based on plasmid or chromosomally mediated resistance mechanisms. Overall, plasmid mediated resistance to either penicillin or tetracycline was exhibited by $175 / 334$ (52\%): 71/334 (21\%) of isolates were 
Table 1 In vitro antimicrobial susceptibility of Neisseria gonorrhoeae: clinical isolates $(\mathbf{n}=334)$

\begin{tabular}{|c|c|c|c|c|c|c|}
\hline \multirow{2}{*}{$\begin{array}{l}\text { Antimicrobial breakpoints } \\
(\text { susceptible/resistant }[\mathrm{mg} / \mathrm{L}])^{\mathrm{a}}\end{array}$} & \multicolumn{3}{|l|}{ No. (\%) } & \multicolumn{3}{|l|}{ MIC $(\mathrm{mg} / \mathrm{L})$} \\
\hline & Susceptible & Intermediate & Resistant & Range & $\mathrm{MIC}_{50}$ & $\mathrm{MIC}_{90}$ \\
\hline Penicillin $G(S \leq 0.06 / R \geq 2)$ & 0 & $108(32.3)$ & $226(67.7)$ & 0.125 to $>8$ & 2 & $>8$ \\
\hline Tetracycline $(S \leq 0.25 / R \geq 2)$ & $2(0.6)$ & $5(1.5)$ & $327(97.9)$ & 0.25 to $>16$ & 8 & $>16$ \\
\hline Ciprofloxacin $(S \leq 0.06 / R \geq 1)$ & 0 & $4(1.2)$ & $330(98.8)$ & $\leq 0.25$ to $>8$ & $>8$ & $>8$ \\
\hline Spectinomycin $(S \leq 32 / R \geq 128)$ & $334(100)$ & 0 & 0 & $\leq 4$ to 32 & 16 & 32 \\
\hline Ceftriaxone $(S \leq 0.25)$ & $334(100)$ & - & - & 0.004 to 0.25 & 0.03 & 0.06 \\
\hline
\end{tabular}

${ }^{a}$ Antimicrobial breakpoints that distinguish susceptible from resistant gonococcal isolates (CLSI Document M07-A9 (M100-S22) [35]. MIC: minimum inhibitory concentration.

PPNG exclusively; 55/334 (16\%) were TRNG exclusively and 49 (15\%) were PPNG/TRNG. CMRNG or TetR alone was identified in $27.8 \%$ and $18.3 \%$ of isolates, respectively. PenR alone was not detected.

\section{Molecular typing of PPNG and TRNG}

$120 \beta$-lactamase producing $N$. gonorrhoeae isolates were distributed into two plasmid types: Asian and African. No Toronto plasmid was detected. 108 (90\%) isolates contained the Asian plasmid and 12 (10\%) harbored the African plasmid.

Among 104 isolates with the tetM determinant, 96.2\% (100/104) carried the Dutch type; the remaining 4 carried the American-type.

The $\beta$-lactamase plasmid was present more frequently in TRNG isolates than in non-TRNG isolates $(47.1 \%$ [49/104] vs. 30.9\% [71/230]; $\mathrm{p}<0.01$; Table 2). Forty nine PPNG/TRNG isolates exhibited two patterns that combined $\beta$-lactamase and tet $M$ containing plasmids: Asian type $\beta$-lactamase expressing isolates always harbored the Dutch tet $M$ type; the combination of African type $\beta$ lactamase and American type tet $M$ was rarely identified in PPNG/TRNG (3 isolates) in Nanjing (Table 2).

\section{Genetic characteristics of $N$. gonorrhoeae isolates with increased MIC to ceftriaxone}

Fifteen (4.5\%) isolates had elevated MICs $(\geq 0.125 \mathrm{mg} / \mathrm{L})$ to ceftriaxone. A second determination on the 15 isolates confirmed the MICs. According to recent CLSI breakpoint criteria [37], these isolates would be classified as "susceptible" but treatment failures have occurred

Table 2 Distribution of $\boldsymbol{\beta}$-lactamase-encoding and tet $M$-encoding plasmids: Neisseria gonorrhoeae clinical isolates $(n=334)$

\begin{tabular}{|c|c|c|c|}
\hline \multirow{2}{*}{$\begin{array}{l}\text { Type of } \\
\text { tet } M \text {-encoding } \\
\text { plasmid }\end{array}$} & \multicolumn{2}{|c|}{$\begin{array}{l}\text { Type of } \beta \text {-lactamase producing } \\
\text { plasmid }\end{array}$} & \multirow[b]{2}{*}{$\begin{array}{l}\beta \text {-lactamase } \\
\text { negative }\end{array}$} \\
\hline & Asian/New Zealand & African/Nîmes & \\
\hline Dutch & 46 & 0 & 54 \\
\hline American & 0 & 3 & 1 \\
\hline tet $M$ negative & 62 & 9 & 159 \\
\hline
\end{tabular}

when infected strains possessed MICs around $0.125 \mathrm{mg} /$ $\mathrm{L}[6,10,11]$. We examined these 15 isolates for genetic mutations in penA, $m$ trR, penB, ponA and pilQ genes (Table 3).

Four PBP2 amino acid sequence patterns were identified; PBP2 allele XVIII was the predominant type $(n=11)$, followed by XIII $(n=2)$ and XII $(n=1)$. A new pattern containing five mutations (345D insertion, A501V, F504L, A510V and A516G) was designated PBP2 allele XLI, according to the previously used nomenclature of PBP2 alleles in $N$. gonorrhoeae [12,15,46-49]; none of these is a mosaic allele. However, 12 isolates (allele XVIII and XLI) possessed an A501T substitution in PBP2, and 2 isolates (allele XIII) had an A501V substitution.

Fourteen of fifteen isolates contained a single nucleotide (A) deletion in the $13 \mathrm{bp}$ inverted repeat motif located between -10 and -35 sequence in the $m t r R$ promoter. Five isolates (including two with ceftriaxone MICs $=0.25 \mathrm{mg} / \mathrm{L}$ ) also had a G45D or A39T substitution in the DNAbinding motif of MtrR, and one isolate possessed a single amino acid replacement (A39T). We did not identify the recently described C-to-T mutation $120 \mathrm{bp}$ upstream of the $m t r C$ start codon, termed mtr $_{120}$ [51].

All isolates possessed penB resistance determinants with substitutions at both G120 and A121 in loop 3 of the PorB1b outer membrane porin; these included G120K/ A121D ( $n=7)$, G120K/A121G $(n=6)$, G120N/A121D $(n=1)$ and $\mathrm{G} 120 \mathrm{~K} / \mathrm{A} 121 \mathrm{~N}(\mathrm{n}=1)$.

Finally, the L421P substitution in PBP1 caused by a single nucleotide mutation ( $\mathrm{T}$ to $\mathrm{C}$ ) in the pon $A$ gene was detected in all isolates; we saw no alternations in the pilQ gene.

The fifteen isolates that harbored 14 different por $B$ sequences and $12 \mathrm{tbpB}$ sequences were assigned to 15 different NG-MAST STs: ST8737, ST3289, ST2186, ST3746, ST2461, ST5061 and 9 new STs. Eleven isolates with the PBP2 allele, XVIII, the most common PBP2, were resolved into 11 separate NG-MAST STs (Table 3 ).

\section{Discussion}

Our study reported high-levels of resistance to previously recommended therapeutic agents for $N$. gonorrhoeae of 
Table 3 Genetic characteristics of Neisseria gonorrhoeae with increased MICs $(n=15)$ to ceftriaxone $(\geq 0.125 \mathrm{mg} / \mathrm{L})$

\begin{tabular}{|c|c|c|c|c|c|c|c|c|c|c|}
\hline \multirow{2}{*}{$\begin{array}{l}\text { Isolate } \\
\text { no. }\end{array}$} & \multirow{2}{*}{$\begin{array}{l}\text { Ceftriaxone } \\
\text { MICs (mg/L) }\end{array}$} & \multirow{2}{*}{$\begin{array}{l}\text { Antimicrobial } \\
\text { characteristics }\end{array}$} & \multicolumn{3}{|c|}{ NG-MAST } & \multirow{2}{*}{$\begin{array}{l}\text { PBP2 } \\
\text { allele }\end{array}$} & \multicolumn{4}{|c|}{ Polymorphisms in: } \\
\hline & & & porB & tbpB & STs & & MtrR & PorB1b & PBP1 & PilQ \\
\hline NJ-1 & 0.25 & CMRNG, CipR & 5658 & 111 & $9530^{*}$ & $X \mathrm{XIII}$ & -57A del, A39T & G120K, A121D & L421P & wt \\
\hline NJ-2 & 0.25 & CMRNG, CipR & 5175 & 328 & 8737 & $X V I I I$ & -57A del, A39T & G120K, A121D & L421P & wt \\
\hline NJ-3 & 0.125 & PPNG, CipR & 5659 & 107 & $9531^{*}$ & $\mathrm{XVIII}$ & $-57 \mathrm{~A}$ del & G120K, A121G & L421P & wt \\
\hline NJ-4 & 0.125 & PP/TRNG, CipR & 1053 & 186 & 3289 & $X V I I I$ & $-57 \mathrm{~A}$ del & G120K, A121D & L421P & wt \\
\hline NJ-5 & 0.125 & PPNG, CipR & 1854 & 33 & $9532^{*}$ & $X L I^{* *}$ & $-57 \mathrm{~A}$ del & G120N, A121D & L421P & wt \\
\hline NJ-6 & 0.125 & PP/TRNG, CipR & 543 & 294 & $9533^{*}$ & $X V I I I$ & A39T & G120K, A121G & L421P & wt \\
\hline NJ-7 & 0.125 & CMRNG, CipR & 5660 & 186 & $9534^{*}$ & $X V I I I$ & $-57 \mathrm{~A}$ del & G120K, A121G & L421P & wt \\
\hline NJ-8 & 0.125 & CMRNG, CipR & 505 & 135 & 2186 & $X V I I I$ & $-57 \mathrm{~A}$ del & G120K, A121D & L421P & wt \\
\hline NJ-9 & 0.125 & CMRNG, CipR & 1285 & 4 & $9535^{*}$ & XIII & $-57 \mathrm{~A}$ del, G45D & G120K, A121D & L421P & wt \\
\hline NJ-10 & 0.125 & TRNG, CipR & 4 & 831 & 3746 & $X I I I$ & $-57 \mathrm{~A}$ del & G120K, A121D & L421P & wt \\
\hline NJ-11 & 0.125 & CMRNG, CipR & 3530 & 4 & $9536^{*}$ & $X \mathrm{XIII}$ & $-57 \mathrm{~A}$ del & G120K, A121G & L421P & wt \\
\hline NJ-12 & 0.125 & CMRNG, CipR & 1198 & 156 & 2461 & $X V I I I$ & -57A del, A39Т & G120K, A121G & L421P & wt \\
\hline NJ-13 & 0.125 & CMRNG, CipR & 5661 & 4 & 5061 & $\mathrm{XVIII}$ & $-57 \mathrm{~A}$ del, G45D & G120K, A121N & L421P & wt \\
\hline NJ-14 & 0.125 & CMRNG, CipR & 2978 & 1058 & $9537^{*}$ & $X \|$ & $-57 \mathrm{~A}$ del & G120K, A121D & L421P & wt \\
\hline NJ-15 & 0.125 & TRNG, CipR & 543 & 438 & $9538^{*}$ & $X V I I I$ & $-57 \mathrm{~A}$ del & G120K, A121G & L421P & wt \\
\hline
\end{tabular}

wt, wild type.

*New NG-MAST STs detected in this study.

${ }^{* *}$ A new PBP2 allele previously not identified was named XLI according to previously used Nomenclature.

\#isolates exhibiting resistance to ciprofloxacin (MIC $\geq 1 \mathrm{mg} / \mathrm{L}$ ).

isolates from men with urethritis in Nanjing (2011-12): ciprofloxacin (98.8\%); tetracycline $(97.9 \%)$ and penicillin $(67.7 \%)$. Rates of resistance have remained unchanged compared with that of 2006 , the last year of a six yearly sequential measurement [39]. Similar patterns of resistance have also been noted from other cities in southern China; Shanghai (ciprofloxacin 98.7\%, tetracycline $73.6 \%$, penicillin $98.1 \%$ [exact time period for isolates collection not available]) [52], Shenzhen (ciprofloxacin 96.1\%, tetracycline $99.0 \%$ in 2008-2012) [53], Hainan (ciprofloxacin 98.0\% in 2011) [54] and other western pacific and southeastern Asian regions including Vietnam, Korea, India, Pakistan and Bhutan [28,31,55-57]. The contribution of plasmid mediated resistance was similar in Nanjing in the period 2011-12 compared to 2006 [39], but during this interval declines in the proportion of PPNG have been observed in Korea [57], Canada [58], Latin America and the Caribbean countries [59]. Because treatment of gonorrhea with ciprofloxacin, tetracycline and penicillin has long been discontinued in Nanjing [30,39], persistence of resistant phenotypes may be explained by the stability of mechanisms that promote resistance or the presence of unidentified selection pressures due to use of antibiotics for other reasons including the common practice of selfmedication with antibiotics. In our study, 33\% of men with gonococcal urethritis reported self-administration of antibiotics in the 30 days prior to their clinic visit (unpublished data).
It is encouraging to note that the percentage of gonococci isolates with decreased susceptibility to ceftriaxone (MIC $\geq 0.125 \mathrm{mg} / \mathrm{L}$ ) declined significantly from $9.1 \%$ (18/ $198)$ in 2006 [39] to $4.5 \%(15 / 334)$ in $2011-12$ ( $\mathrm{p}=0.04)$; a one dilution decrease of the modal MIC for ceftriaxone was also observed $(0.06 \mathrm{mg} / \mathrm{L}$ in 2006 to $0.03 \mathrm{mg} / \mathrm{L}$ in 2011-12) (Figure 1). In Europe [32,60-63], Australia [64], Africa [65-67], the western hemisphere [33,58,59,68-70] and the South-East Asia Region [31], MICs to ceftriaxone have risen during this period. Nonetheless, it is of concern that the distribution of spectinomycin MICs shifted upward in Nanjing during the same interval, between 2006 and 2011-12 (Figure 2) [39]. In 2006, an MIC = $32 \mathrm{mg} / \mathrm{L}$ to spectinomycin was observed in $1.0 \%$ of strains, rising to $18.3 \%$ in $2011-12(\mathrm{p}<0.01)$, coinciding with a significant decrease in the percentage of strains whose MICs $\leq 8 \mathrm{mg} / \mathrm{L}$ $(\mathrm{p}<0.01)$. Opposing trends in resistance of gonococcal isolates to ceftriaxone and spectinomycin may, in part, result from the greater use of spectinomycin in the Nanjing STD clinic. Ninety-eight percent of gonococcal urethritis cases in men were prescribed spectinomycin as recently as in 2012, in large part because of the lower price of Chinese-manufactured spectinomycin vs. imported ceftriaxone and the requirements for cefriaxone skin testing prior to administration. Resistance of $N$. gonorrhoeae to spectinomycin, although rarely reported $[31,33,65,71]$, because of limited access to and use of this antibiotic in many countries outside of China, may be the "cautionary 


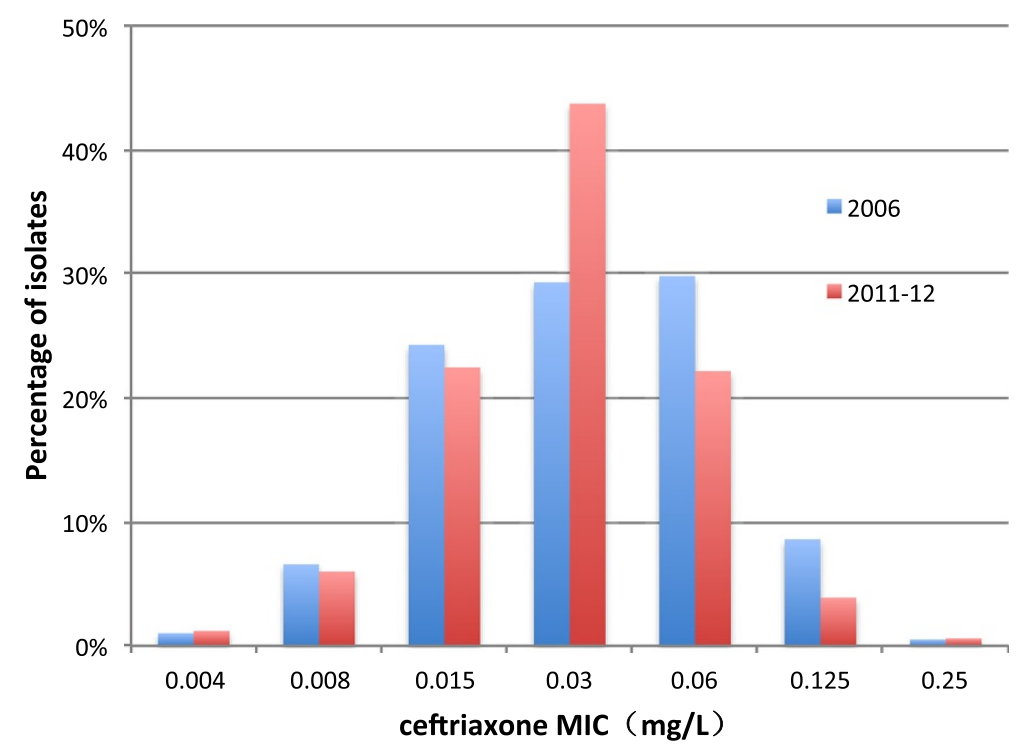

Figure 1 Distribution of ceftriaxone MICs of Neisseria gonorrhoeae isolates: Nanjing, $2006(n=198)$ and 2011-12 ( $n=334)$.

tale" of clinical failure when it is used as primary treatment of gonorrhea [72].

Plasmids carried by PPNG and TRNG isolates have changed over time in Nanjing [39]. The highly active TEM $\beta$-lactamase gene is carried by a family of plasmids in N. gonorrhoeae. Since 1976, at least eight different plasmids have been identified from geographically distinct locations [73]. The $\beta$-lactamase gene has been found on Asian-, African- and Toronto - type plasmids carried by epidemic strains of $N$. gonorrhoeae [74], even while new types such as the Johannesburg and Australian plasmids are being discovered $[75,76]$. We did not differentiate the
Nîmes plasmid from the African-type or the New Zealand plasmid from the Asian-type, owing to the identical sizes of the PCR products but we would have identified the novel Johannesburg plasmid identified by the 458 bp PCR products using the primer pair designed for the Africantype plasmid. Using a simple multiplex PCR method [43], we detected either Asian/ New Zealand type $(n=108)$ or African/Nîmes type $(n=12)$ plasmids in all penicillinasepositive isolates. The Asian/New Zealand type plasmid dominated, while the newly emerging African/Nimes type, which was absent in Nanjing isolates of 2006, and were epidemic in the United Kingdom (UK) and West Africa

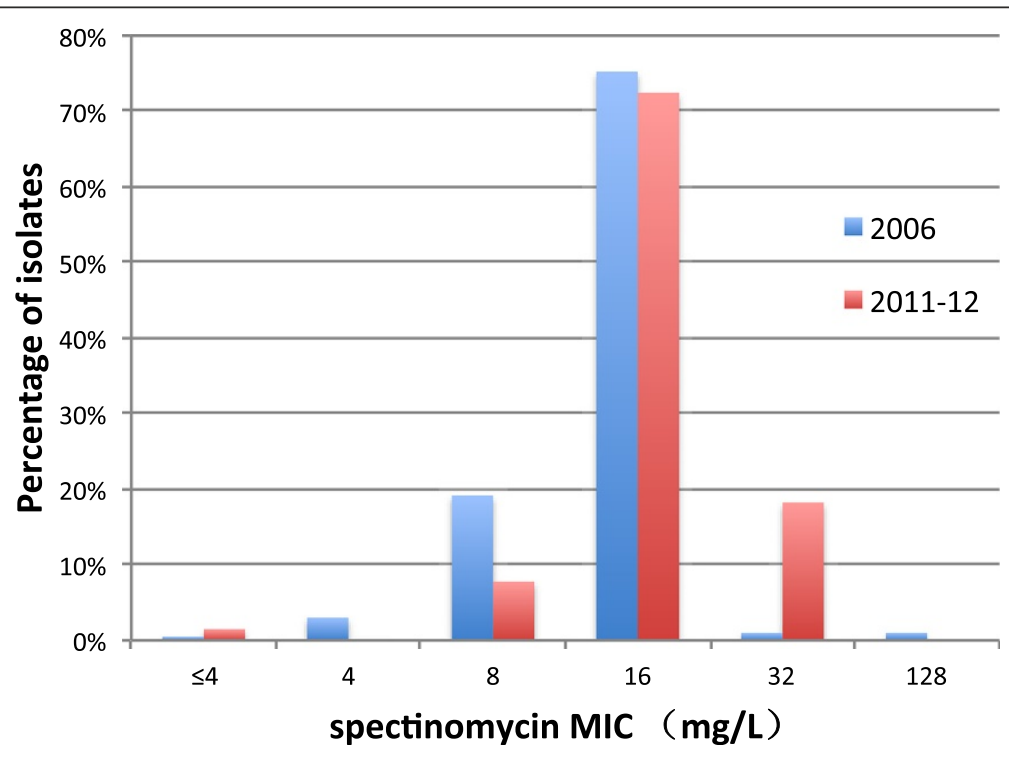

Figure 2 Distribution of spectinomycin MICs of Neisseria gonorrhoeae isolates: Nanjing, $2006(n=198)$ and $2011-12(n=334)$. 
[74], may have reflected movement of $N$. gonorrhoeae across continents. In like manner, while the Dutch variant $(\mathrm{n}=100)$ of tetM still predominates, identification of the minor American variant $(n=4)$ in Nanjing is just beginning.

Decreased susceptibility to ceftriaxone was demonstrated in $15(4.5 \%)$ of the Nanjing isolates of N. gonorrhoeae (cefixime was not tested/not used in Nanjing). Differing from gonococcal isolates that were resistant or less susceptible to ESCs from Japan [77], Europe $[10,38,78-80]$, North America $[47,81]$ or South Africa [67], the mosaic-like structure that is usually located in the region of the transpeptidase-encoding domain of penA of resistant gonococcal strains, was not identified in the Nanjing isolates. Similar to strains recently tested from Southeast Asia (Vietnam) [56] and South Asia [55], the majority of our 15 isolates with decreased susceptibility contained mutations in PBP2 at position 501: A501V or A501T. Whiley et al. [13] have suggested that gonococcal-specific A501 substitutions may contribute more significantly to reduced ceftriaxone susceptibility than mosaic sequences. Three A501 mutation-containing patterns of PBP2 (XVIII, XIII and $\mathrm{XLI}$ ) in our study have been reported to be epidemiologically connected with increased MICs to ceftriaxone $[15,44,45]$. The results of a modeling study indicated that reduced susceptibility to cephems such as cefixime and ceftriaxone is due to a conformational alteration of the beta-lactam-binding pocket caused by an A501 mutation in non-mosaic strains [13]. Results of the model have been confirmed by isogenic transformation demonstrating, that an $\mathrm{A} 501 \mathrm{~V}(\mathrm{~T})$ mutation in PBP2 results in increased MIC to ceftriaxone in a non-mosaic penA containing strain [82]. Polymorphisms in $m t r R$ and penB genes (and also pon $A$ ) were also evident in our isolates.

Azithromycin, combined with ceftriaxone, recently has been recommended by the United States [83] and the United Kingdom [84] and is now also in the European guidelines [85] for the treatment uncomplicated gonorrhea. This combination had not been recommended for the treatment of gonorrhea in China during the study period (2011-2012). Azithromycin resistance of $N$. gonorhoeae isolates from the Nanjing STD clinic was 6.8\% [86] in 2008 and 2009, which exceeded the 5\% threshold suggested by WHO for emperic use as a first line agent [26-29].

Our study has several limitations. Gonococcal isolates were collected only from men with symptomatic urethritis. 33\% of infected men had used antibiotics prior to their clinic visit, which may have contributed to increased resistance of their gonococcal isolates. Exclusion of isolates from asymptomatic men and from extragential sites (there were only two MSMs in our study) may have underestimated AMR [87-89]. Simultaneously, infected men may have been cured of infection with isolates sensitive to the antibiotics that they had used earlier and therefore not seen in the clinic. This may have also led to an overabundance of more resistant $N$. gonorrhoeae.

\section{Conclusions}

This study confirmed that ceftriaxone and spectinomycin remain effective empiric first-line therapy for gonorrhea in Nanjing. However, years of discontinued use of formerly recommended antimicrobial agents did not restore the sensitivities of gonococcal strains to ciprofloxacin, penicillin or tetracycline. Changes and multiple representative types of resistance plasmid profiles highlight frequent influx and exchange of $N$. gonorrhoeae strains. Reduced ceftriaxone susceptibility was found in $4.5 \%$ of gonococcal isolates and alterations in penA, $m t r R$, and penB genes were important determinants of resistance. Sustained surveillance of gonococcal phenotypic and genotypic AMR is an important element in the control of gonoccocal infection.

\section{Abbreviations}

AMR: Antimicrobial resistance; PPNG: Penicillinase-producing N. gonorrhoeae; TRNG: Tetracycline-resistant N. gonorrhoeae; MIC: Minimum inhibitory concentration; NG-MAST: Neisseria gonorrhoeae multi-antigen sequence typing; ST: Sequence type; STI: Sexually transmitted infection; STD: Sexually transmitted diseases; ESC: Extended-spectrum cephalosporin; WHO: World Health Organization; CLSI: Clinical and Laboratory Standards Institute; PBP: Penicillin-binding protein.

\section{Competing interests}

The authors declare no competing interests.

\section{Authors' contributions}

SL completed the gene determinants sequencing, NG-MAST, assisted with the antimicrobial susceptibility testing and prepared the manuscript. XHS directed the project, supervised the bacterial isolates collection and consulted on manuscript preparation. WJL and FXJ carried out the antimicrobial susceptibility testing and the tet $M$ and $\beta$-lactamase gene producing plasmids identification. BXW and PAR consulted on the project and manuscript preparation. All authors read and approved the final manuscript.

\section{Acknowledgements}

This work was supported in part by NIH grant Al084048.

\section{Author details}

${ }^{1}$ STD Clinic, Institute of Dermatology, Chinese Academy of Medical Sciences and Peking Union Medical College, Nanjing 210042, China. ${ }^{2}$ Department of Dermatology, Anhui Provincial Hospital, Hefei 230001, China. ${ }^{3}$ Division of Infectious Diseases and Immunology, University of Massachusetts Medical School, Worcester, MA 01602, USA.

Received: 4 October 2014 Accepted: 7 November 2014

Published online: 27 November 2014

\section{References}

1. National Health and Family Planning Commision of the People's Republic of China: 2013 Annual National Report Of Notifiable Infectious Diseases [http://www.nhfpc.gov.cn/jkj/s3578/201404/f81a87a2fe3f453a99677a7 d9d89de0c]

2. World Health Organization $(\mathrm{WHO})$ Department of Reproductive Health and Research: Global action Plan to Control the Spread and Impact of Antimicrobial Resistance in Neisseria Gonorrhoeae. Geneva: WHO Press; 2012 [http://whqlibdoc.who.int/publications/2012/9789241503501_eng.pdf] 
3. Tapsall J: Antibiotic resistance in Neisseria gonorrhoeae is diminishing available treatment options for gonorrhea: some possible remedies. Expert Rev Anti Infect Ther 2006, 4:619-628.

4. Unemo M, Shafer WM: Antibiotic resistance in Neisseria gonorrhoeae: origin, evolution, and lessons learned for the future. Ann N Y Acad Sci 2011, 1230:E19-E28.

5. Ison CA, Hussey J, Sankar KN, Evans J, Alexander S: Gonorrhoea treatment failures to cefixime and azithromycin in England, 2010. Euro Surveill 2011, 16(14).

6. Unemo M, Nicholas RA: Emergence of multidrug-resistant, extensively drug-resistant and untreatable gonorrhea. Future Microbio/ 2012, 7:1401-1422.

7. Barry PM, Klausner JD: The use of cephalosporins for gonorrhea: the impending problem of resistance. Expert Opin Pharmacother 2009, 10:555-577.

8. Unemo M, Golparian D, Syversen G, Vestrheim DF, Moi H: Two cases of verified clinical failures using internationally recommended first-line cefixime for gonorrhoea treatment, Norway, 2010. Euro Surveill 2010, 15(47).

9. Ohnishi M, Golparian D, Shimuta K, Saika T, Hoshina S, Iwasaku K, Nakayama S, Kitawaki J, Unemo M: Is Neisseria gonorrhoeae initiating a future era of untreatable gonorrhea?: detailed characterization of the first strain with high-level resistance to ceftriaxone. Antimicrob Agents Chemother 2011, 55:3538-3545

10. Unemo M, Golparian D, Potocnik M, Jeverica S: Treatment failure of pharyngeal gonorrhoea with internationally recommended first-line ceftriaxone verified in Slovenia, September 2011. Euro Surveill 2012, 17(25).

11. Tapsall J, Read P, Carmody C, Bourne C, Ray S, Limnios A, Sloots T, Whiley D: Two cases of failed ceftriaxone treatment in pharyngeal gonorrhoea verified by molecular microbiological methods. J Med Microbiol 2009, 58:683-687.

12. Ito M, Deguchi T, Mizutani KS, Yasuda M, Yokoi S, Ito S, Takahashi Y, Ishihara S, Kawamura Y, Ezaki T: Emergence and spread of Neisseria gonorrhoeae clinical isolates harboring mosaic-like structure of penicillin-binding protein 2 in Central Japan. Antimicrob Agents Chemother 2005, 49:137-143.

13. Osaka K, Takakura T, Narukawa K, Takahata M, Endo K, Kiyota H, Onodera S: Analysis of amino acid sequences of penicillin-binding protein 2 in clinical isolates of Neisseria gonorrhoeae with reduced susceptibility to cefixime and ceftriaxone. J Infect Chemother 2008, 14:195-203.

14. Powell AJ, Tomberg J, Deacon AM, Nicholas RA, Davies C: Crystal structures of penicillin-binding protein 2 from penicillin-susceptible and -resistant strains of Neisseria gonorrhoeae reveal an unexpectedly subtle mechanism for antibiotic resistance. J Biol Chem 2009, 284:1202-1212

15. Whiley DM, Limnios EA, Ray S, Sloots TP, Tapsall JW: Diversity of penA alterations and subtypes in Neisseria gonorrhoeae strains from Sydney, Australia, that are less susceptible to ceftriaxone. Antimicrob Agents Chemother 2007, 51:3111-3116.

16. Whiley DM, Goire N, Lambert SB, Ray S, Limnios EA, Nissen MD, Sloots TP, Tapsall JW: Reduced susceptibility to ceftriaxone in Neisseria gonorrhoeae is associated with mutations G542S, P551S and P551L in the gonococcal penicillin-binding protein 2. J Antimicrob Chemother 2010, 65:1615-1618.

17. Warner DM, Shafer WM, Jerse AE: Clinically relevant mutations that cause derepression of the Neisseria gonorrhoeae MtrC-MtrD-MtrE Efflux pump system confer different levels of antimicrobial resistance and in vivo fitness. Mol Microbiol 2008, 70:462-478.

18. Hagman KE, Pan W, Spratt BG, Balthazar JT, Judd RC, Shafer WM: Resistance of Neisseria gonorrhoeae to antimicrobial hydrophobic agents is modulated by the mtrRCDE efflux system. Microbiology 1995, 141(Pt 3):611-622.

19. Gill MJ, Simjee S, Al-Hattawi K, Robertson BD, Easmon CS, Ison CA: Gonococcal resistance to beta-lactams and tetracycline involves mutation in loop 3 of the porin encoded at the penB locus. Antimicrob Agents Chemother 1998, 42:2799-2803.

20. Ropp PA, Hu M, Olesky M, Nicholas RA: Mutations in ponA, the gene encoding penicillin-binding protein 1 , and a novel locus, penC, are required for high-level chromosomally mediated penicillin resistance in Neisseria gonorrhoeae. Antimicrob Agents Chemother 2002, 46:769-777.

21. Zhao S, Tobiason DM, Hu M, Seifert HS, Nicholas RA: The penC mutation conferring antibiotic resistance in Neisseria gonorrhoeae arises from a mutation in the PilQ secretin that interferes with multimer stability. Mol Microbiol 2005, 57:1238-1251.
22. Whiley DM, Jacobsson S, Tapsall JW, Nissen MD, Sloots TP, Unemo M: Alterations of the pilQ gene in Neisseria gonorrhoeae are unlikely contributors to decreased susceptibility to ceftriaxone and cefixime in clinical gonococcal strains. J Antimicrob Chemother 2010, 65:2543-2547.

23. Zhao S, Duncan M, Tomberg J, Davies C, Unemo M, Nicholas RA: Genetics of chromosomally mediated intermediate resistance to ceftriaxone and cefixime in Neisseria gonorrhoeae. Antimicrob Agents Chemother 2009, 53:3744-3751.

24. Centers for Disease Control and Prevention (CDC): Cephalosporin-Resistant Neisseria Gonorrhoeae Public Health Response Plan. CDC 2012 [http://www.cdc.gov/std/treatment/Ceph-R-ResponsePlanJuly30-2012.pdf]

25. European Centre for Disease Prevention and Control (ECDC): Response Plan to Control and Manage the Threat of Multidrug-Resistant Gonorrhoea in Europe. Stockholm: ECDC; 2012.

26. National Center for STD Control, China CDC: Brief report of Chinese Gonococcal Surveillance. [http://www.ncstdc.org/show.asp?id=967]

27. W. H. O. Western Pacific South East Asian Gonococcal Antimicrobial Surveillance, Programmes: Surveillance of antibiotic resistance in Neisseria gonorrhoeae in the WHO Western Pacific and South East Asian Regions, 2009. Commun Dis Intell Q Rep 2011, 35:2-7.

28. Lahra MM, WHO Western Pacific South East Asian Gonococcal Antimicrobial Surveillance Programme: Surveillance of antibiotic resistance in Neisseria gonorrhoeae in the WHO Western Pacific and South East Asian Regions, 2010. Commun Dis Intell Q Rep 2012, 36:95-100.

29. WHO Western Pacific Region Gonococcal Antimicrobial Surveillance Programme: Surveillance of antibiotic susceptibility of Neisseria gonorrhoeae in the WHO western Pacific region 1992-4. Genitourin Med 1997, 73:355-361.

30. National Center for STD Control, China CDC: Gonorrhea. In Guidelines for Diagnosis and Treatment of Sexually Transmitted Diseases [in Chinese]. Edited by Wang QQ, Zhang GC. Shanghai: Shanghai Scientific and Technological Literature Press; 2007

31. Bala M, Kakran M, Singh V, Sood S, Ramesh V, Members of WHOGSN: Monitoring antimicrobial resistance in Neisseria gonorrhoeae in selected countries of the WHO South-East Asia Region between 2009 and 2012: a retrospective analysis. Sex Transm Infect 2013, 89(4):iv28-iv35.

32. Ison CA, Town K, Obi C, Chisholm S, Hughes G, Livermore DM, Lowndes CM, GRASP collaborative group: Decreased susceptibility to cephalosporins among gonococci: data from the Gonococcal Resistance to Antimicrobials Surveillance Programme (GRASP) in England and Wales, 2007-2011. Lancet Infect Dis 2013, 13:762-768.

33. Kirkcaldy RD, Kidd S, Weinstock HS, Papp JR, Bolan GA: Trends in antimicrobial resistance in Neisseria gonorrhoeae in the USA: the Gonococcal Isolate Surveillance Project (GISP), January 2006-June 2012. Sex Transm Infect 2013, 89(4):iv5-iv10.

34. World Health Organization: Antimicrobial Resistance Global Report on Surveillance: 2014 Summary. Geneva: WHO Press; 2014 [http://apps.who.int/ iris/bitstream/10665/112647/1/WHO_HSE_PED_AIP_2014.2_eng.pdf]

35. Tapsall J: Antimicrobial Resistance in Neisseria Gonorrhoeae. Geneva: WHO Press; 2001 [http://apps.who.int/iris/bitstream/10665/66963/1/WHO_ CDS_CSR_DRS_2001.3.pdf]

36. Sherrard J, Barlow D: Gonorrhoea in men: clinical and diagnostic aspects. Genitourin Med 1996, 72:422-426.

37. CLSI: Neisseria gonorrhoeae. In Performance Standards for Antimicrobial Susceptibility Testing; Twenty-Second Informational Supplement. CLSI document M100-S22. Wayne, PA: Clinical and Laboratory Standards Institute; 2012:100-102.

38. Camara J, Serra J, Ayats J, Bastida T, Carnicer-Pont D, Andreu A, Ardanuy C: Molecular characterization of two high-level ceftriaxone-resistant Neisseria gonorrhoeae isolates detected in Catalonia, Spain. J Antimicrob Chemother 2012, 67:1858-1860

39. Su X, Jiang F, Qimuge, Dai X, Sun H, Ye S: Surveillance of antimicrobial susceptibilities in Neisseria gonorrhoeae in Nanjing, China, 1999-2006. Sex Transm Dis 2007, 34:995-999.

40. Sng EH, Yeo KL, Rajan VS: Simple method for detecting penicillinaseproducing Neisseria gonorrhoeae and Staphylococcus aureus. Br J Vener Dis 1981, 57:141-142.

41. Gorwitz RJ, Nakashima AK, Moran JS, Knapp JS: Sentinel surveillance for antimicrobial resistance in Neisseria gonorrhoeae-United States, 19881991. The Gonococcal Isolate Surveillance Project Study Group. MMWR CDC Surveill Summ 1993, 42:29-39. 
42. Turner A, Gough KR, Leeming JP: Molecular epidemiology of tetM genes in Neisseria gonorrhoeae. Sex Transm Infect 1999, 75:60-66.

43. Palmer HM, Leeming JP, Turner A: A multiplex polymerase chain reaction to differentiate beta-lactamase plasmids of neisseria gonorrhoeae. J Antimicrob Chemother 2000, 45:777-782.

44. Liao M, Gu WM, Yang Y, Dillon JA: Analysis of mutations in multiple loci of Neisseria gonorrhoeae isolates reveals effects of PIB, PBP2 and MtrR on reduced susceptibility to ceftriaxone. J Antimicrob Chemother 2011, 66:1016-1023

45. Lee SG, Lee H, Jeong SH, Yong D, Chung GT, Lee YS, Chong Y, Lee K: Various pen $A$ mutations together with $\mathrm{mtrR}$, porB and ponA mutations in Neisseria gonorrhoeae isolates with reduced susceptibility to cefixime or ceftriaxone. J Antimicrob Chemother 2010, 65:669-675.

46. Ohnishi M, Watanabe Y, Ono E, Takahashi C, Oya H, Kuroki T, Shimuta K, Okazaki N, Nakayama S, Watanabe H: Spread of a chromosomal cefiximeresistant penA gene among different Neisseria gonorrhoeae lineages. Antimicrob Agents Chemother 2010, 54:1060-1067.

47. Martin I, Sawatzky P, Allen V, Hoang L, Lefebvre B, Mina N, Wong T, Gilmour $M$ : Emergence and characterization of Neisseria gonorrhoeae isolates with decreased susceptibilities to ceftriaxone and cefixime in Canada: 2001-2010. Sex Transm Dis 2012, 39:316-323.

48. Chen CC, Lin KY, Li SY: The role of penicillin-binding protein 2 (PBP2) in the cephalosporin susceptibility of Neisseria gonorrhoeae and the need for consensus in naming of PBP2. J Formos Med Assoc 2012, 111:665-666.

49. Allen VG, Farrell DJ, Rebbapragada A, Tan J, Tijet N, Perusini SJ, Towns L, Lo S, Low DE, Melano RG: Molecular analysis of antimicrobial resistance mechanisms in Neisseria gonorrhoeae isolates from Ontario, Canada. Antimicrob Agents Chemother 2011, 55:703-712.

50. Martin IM, Ison CA, Aanensen DM, Fenton KA, Spratt BG: Rapid sequencebased identification of gonococcal transmission clusters in a large metropolitan area. J Infect Dis 2004, 189:1497-1505.

51. Ohneck EA, Zalucki YM, Johnson PJ, Dhulipala V, Golparian D, Unemo M, Jerse AE, Shafer WM: A novel mechanism of high-level, broad-spectrum antibiotic resistance caused by a single base pair change in Neisseria gonorrhoeae. MBio 2011, 2:e00187-11.

52. Yang Y, WL ZC, Gu W: Study of antimicrobial susceptibilities and molecular resistance determines in Neisseria gonorrhoeae isolated in Shanghai. Chin J Lab Med 2009, 32:1173-1175.

53. Jiang $Y, L J X Y$, Lin $H L$, Wang $Y X$ : Study on drug resistance of 103 strains of Neisseria gonorrhoeae. Practical Preventive Medicine 2013, 20:345-347.

54. Zhong N, ZW WF, Zhang LF, Lu YZ: Analysis on the drug-resistance of Nersseria gonorrhoeae to antibiotics in Hainan area from 2006-2011. Chin J Derm Venereol 2013, 27:56-57.

55. Sethi S, Golparian D, Bala M, Dorji D, Ibrahim M, Jabeen K, Unemo M: Antimicrobial susceptibility and genetic characteristics of Neisseria gonorrhoeae isolates from India, Pakistan and Bhutan in 2007-2011. BMC Infect Dis 2013, 13:35.

56. Olsen B, Pham TL, Golparian D, Johansson E, Tran HK, Unemo M: Antimicrobial susceptibility and genetic characteristics of Neisseria gonorrhoeae isolates from Vietnam, 2011. BMC Infect Dis 2013, 13:40.

57. Lee H, Hong SG, Soe Y, Yong D, Jeong SH, Lee K, Chong Y: Trends in antimicrobial resistance of Neisseria gonorrhoeae isolated from Korean patients from 2000 to 2006. Sex Transm Dis 2011, 38:1082-1086.

58. Martin I, Jayaraman G, Wong T, Liu G, Gilmour M, Canadian Public Health Laboratory Network: Trends in antimicrobial resistance in Neisseria gonorrhoeae isolated in Canada: 2000-2009. Sex Transm Dis 2011, 38:892-898.

59. Dillon JA, Trecker MA, Thakur SD, Gonococcal Antimicrobial Surveillance Program Network in Latin A, Caribbean: Two decades of the gonococcal antimicrobial surveillance program in South America and the Caribbean: challenges and opportunities. Sex Transm Infect 2013, 89(4):iv36-iv41.

60. Kubanova A, Frigo N, Kubanov A, Sidorenko S, Lesnaya I, Polevshikova S, Solomka V, Bukanov N, Domeika M, Unemo M: The Russian gonococcal antimicrobial susceptibility programme (RU-GASP)-national resistance prevalence in 2007 and 2008, and trends during 2005-2008. Euro Surveill 2010, 15(14)

61. Cole MJ, Unemo M, Hoffmann S, Chisholm SA, Ison CA, van de Laar MJ: The European gonococcal antimicrobial surveillance programme, 2009. Euro Surveill 2011, 16(42)

62. Cole MJ, Chisholm SA, Hoffmann S, Stary A, Lowndes CM, Ison CA European Surveillance of Sexually Transmitted Infections Network: European surveillance of antimicrobial resistance in Neisseria gonorrhoeae. Sex Transm Infect 2010, 86:427-432

63. European Centre for Disease Prevention and Control: Gonococcal Antimicrobial Susceptibility Surveillance in Europe 2011. Stockholm: ECDC; 2013.

64. Australian Gonococcal Surveillance P: Australian Gonococcal Surveillance Programme annual report, 2010. Commun Dis Intell Q Rep 2011, 35:229-236.

65. Ndowa FJ, Francis JM, Machiha A, Faye-Kette H, Fonkoua MC: Gonococcal antimicrobial resistance: perspectives from the African region. Sex Transm Infect 2013, 89(4):iv11-iv15.

66. Mehta SD, Maclean I, Ndinya-Achola JO, Moses S, Martin I, Ronald A, Agunda L, Murugu R, Bailey RC, Melendez J, Zenilman JM: Emergence of quinolone resistance and cephalosporin MIC creep in Neisseria gonorrhoeae isolates from a cohort of young men in Kisumu, Kenya, 2002 to 2009. Antimicrob Agents Chemother 2011, 55:3882-3888.

67. Lewis DA, Sriruttan C, Muller EE, Golparian D, Gumede L, Fick D, de Wet J, Maseko V, Coetzee J, Unemo M: Phenotypic and genetic characterization of the first two cases of extended-spectrum-cephalosporin-resistant Neisseria gonorrhoeae infection in South Africa and association with cefixime treatment failure. J Antimicrob Chemother 2013, 68:1267-1270.

68. Martin I, Sawatzky P, Liu G, Allen V, Lefebvre B, Hoang L, Lovgren M, Haldane D, Caeseele PV, Horsman G, Garceau R, Ratnam S, Wong T, Gilmour M: Antimicrobial susceptibilities and distribution of sequence types of Neisseria gonorrhoeae isolates in Canada: 2010. Can J Microbiol 2013, 59:671-678.

69. Hottes TS, Lester RT, Hoang LM, McKay R, Imperial M, Gilbert M, Patrick D, Wong T, Martin I, Ogilvie G: Cephalosporin and azithromycin susceptibility in Neisseria gonorrhoeae isolates by site of infection, British Columbia, 2006 to 2011. Sex Transm Dis 2013, 40:46-51.

70. Centers for Disease Control and Prevention: Cephalosporin susceptibility among Neisseria gonorrhoeae isolates-United States, 2000-2010. MMWR Morb Mortal Wkly Rep 2011, 60:873-877

71. Unemo M, Golparian D, Skogen V, Olsen AO, Moi H, Syversen G, Hjelmevoll SO: Neisseria gonorrhoeae strain with high-level resistance to spectinomycin due to a novel resistance mechanism (mutated ribosomal protein S5) verified in Norway. Antimicrob Agents Chemother 2013, 57:1057-1061.

72. Boslego JW, Tramont EC, Takafuji ET, Diniega BM, Mitchell BS, Small JW, Khan WN, Stein DC: Effect of spectinomycin use on the prevalence of spectinomycin-resistant and of penicillinase-producing Neisseria gonorrhoeae. N Engl J Med 1987, 317:272-278.

73. Phillips I: Beta-lactamase-producing, penicillin-resistant gonococcus. Lancet 1976, 2:656-657.

74. Dillon JA, Yeung KH: Beta-lactamase plasmids and chromosomally mediated antibiotic resistance in pathogenic Neisseria species. Clin Microbiol Rev 1989, 2(Suppl):S125-S133.

75. Fayemiwo SA, Muller EE, Gumede L, Lewis DA: Plasmid-mediated penicillin and tetracycline resistance among Neisseria gonorrhoeae isolates in South Africa: prevalence, detection and typing using a novel molecular assay. Sex Transm Dis 2011, 38:329-333.

76. Trembizki E, Buckley C, Lawrence A, Lahra M, Whiley D, GRAND Study Investigators: Characterisation of a novel Neisseria gonorrhoeae penicillinase-producing plasmid isolated in Australia in 2012. Antimicrob Agents Chemother 2014, 58:4984-4985.

77. Shimuta K, Unemo M, Nakayama S, Morita-Ishihara T, Dorin M, Kawahata T, Ohnishi M, Antibiotic-Resistant Gonorrhea Study Group: Antimicrobial resistance and molecular typing of Neisseria gonorrhoeae isolates in Kyoto and Osaka, Japan, 2010 to 2012: intensified surveillance after identification of the first strain ( $\mathrm{H} 041)$ with high-level ceftriaxone resistance. Antimicrob Agents Chemother 2013, 57:5225-5232.

78. Unemo M, Golparian D, Nicholas R, Ohnishi M, Gallay A, Sednaoui P: Highlevel cefixime- and ceftriaxone-resistant Neisseria gonorrhoeae in France: novel penA mosaic allele in a successful international clone causes treatment failure. Antimicrob Agents Chemother 2012, 56:1273-1280.

79. Hjelmevoll SO, Golparian D, Dedi L, Skutlaberg DH, Haarr E, Christensen A, Jorgensen S, Nilsen OJ, Unemo M, Skogen V: Phenotypic and genotypic properties of Neisseria gonorrhoeae isolates in Norway in 2009: antimicrobial resistance warrants an immediate change in national management guidelines. Eur J Clin Microbiol Infect Dis 2012, 31:1181-1186

80. Golparian D, Hellmark B, Fredlund H, Unemo M: Emergence, spread and characteristics of Neisseria gonorrhoeae isolates with in vitro decreased 
susceptibility and resistance to extended-spectrum cephalosporins in Sweden. Sex Transm Infect 2010, 86:454-460.

81. Gose S, Nguyen D, Lowenberg D, Samuel M, Bauer H, Pandori M: Neisseria gonorrhoeae and extended-spectrum cephalosporins in California: surveillance and molecular detection of mosaic penA. BMC Infect Dis 2013, 13:570.

82. Tomberg J, Unemo M, Davies C, Nicholas RA: Molecular and structural analysis of mosaic variants of penicillin-binding protein 2 conferring decreased susceptibility to expanded-spectrum cephalosporins in Neisseria gonorrhoeae: role of epistatic mutations. Biochemistry 2010, 49:8062-8070.

83. Centers for Disease Control Prevention: Update to CDC's sexually transmitted diseases treatment guidelines, 2010: oral cephalosporins no longer a recommended treatment for gonococcal infections. MMWR Morb Mortal Wkly Rep 2012, 61:590-594.

84. Bignell C, Fitzgerald M, Guideline Development Group, British Association for Sexual Health and HIV UK: UK national guideline for the management of gonorrhoea in adults, 2011. Int J STD AIDS 2011, 22:541-547.

85. Bignell C, Unemo M, European STI Guidelines Editorial Board: 2012 European guideline on the diagnosis and treatment of gonorrhoea in adults. Int J STD AIDS 2013, 24:85-92.

86. Yuan LF, Yin YP, Dai XQ, Pearline RV, Xiang Z, Unemo M, Chen XS: Resistance to azithromycin of Neisseria gonorrhoeae isolates from 2 cities in China. Sex Transm Dis 2011, 38:764-768.

87. Read PJ, Limnios EA, McNulty A, Whiley D, Lahra MM: One confirmed and one suspected case of pharyngeal gonorrhoea treatment failure following $500 \mathrm{mg}$ ceftriaxone in Sydney, Australia. Sex Health 2013, 10:460-462.

88. YC M, Stevens K, Tideman R, Zaia A, Tomita T, Fairley CK, Lahra M, Whiley D, Hogg G: Failure of $500 \mathrm{mg}$ of ceftriaxone to eradicate pharyngeal gonorrhoea, Australia. J Antimicrob Chemother 2013, 68:1445-1447.

89. Deguchi $T$, Yasuda $M$, Ito $\mathrm{S}$ : Management of pharyngeal gonorrhea is crucial to prevent the emergence and spread of antibiotic-resistant Neisseria gonorrhoeae. Antimicrob Agents Chemother 2012, 56:4039-4040. author reply 4041-4032.

doi:10.1186/s12879-014-0622-0

Cite this article as: Li et al:: Antimicrobial susceptibility of Neisseria gonorrhoeae isolates from symptomatic men attending the Nanjing sexually transmitted diseases clinic (2011-2012): genetic characteristics of isolates with reduced sensitivity to ceftriaxone. BMC Infectious Diseases 2014 14:622.

\section{Submit your next manuscript to BioMed Central and take full advantage of:}

- Convenient online submission

- Thorough peer review

- No space constraints or color figure charges

- Immediate publication on acceptance

- Inclusion in PubMed, CAS, Scopus and Google Scholar

- Research which is freely available for redistribution 